Research Article

\title{
Research on Feature Fusion Method of Mine Microseismic Signal Based on Unsupervised Learning
}

\author{
Rui Liu \\ School of Energy and Mining Engineering, China University of Mining and Technology (Beijing), Beijing 100083, China \\ Correspondence should be addressed to Rui Liu; bukaopu999@gmail.com
}

Received 19 June 2021; Accepted 13 September 2021; Published 6 October 2021

Academic Editor: Arturo Garcia-Perez

Copyright (c) 2021 Rui Liu. This is an open access article distributed under the Creative Commons Attribution License, which permits unrestricted use, distribution, and reproduction in any medium, provided the original work is properly cited.

\begin{abstract}
The feature extraction of high-precision microseismic signals is an important prerequisite for multicategory recognition of microseismic signals, and it is also an important basis for intelligent sensing modules in smart mines. Aiming at the problem of unobvious feature extraction of multiclass mine microseismic signals, this paper is based on the unsupervised learning method in the deep learning method, combined with wavelet packet energy ratio and empirical modulus singular value decomposition, and proposes a method based on wavelet packet energy and empirical modulus singular value decomposition and proposes a method (M-W\&E) based on wavelet packet energy and empirical modulus singular value decomposition. This method firstly performs empirical modulus singular value decomposition and wavelet packet energy ratio on the microseismic signal to construct the basic feature vector and then uses the unsupervised learning algorithm to perform the unsupervised learning method feature fusion of the basic feature vector to construct the fused feature vector. After visualization by t-SNE, various distinctions in the fusion feature vector are more obvious. After testing the fusion feature classification using SVM, it is found that the recognition rate of the new feature after feature fusion is better than that of a single wavelet packet empirical energy component and singular value of empirical modulus, which basically meets the engineering needs and is a mine microseism. The signal extraction and feature enhancement fusion of multiclass samples provide a new idea.
\end{abstract}

\section{Introduction}

With the substantial development of the mathematics industry and the computer industry, there has been some progress in processing time series data $[1,2]$. As a new method of monitoring the state of rocks in recent years, the microseismic monitoring system has been used in mines, tunnels, petroleum, and so on. The field has been applied [3-8], and at the same time, more fruitful results have been achieved, realizing the prediction and forecasting of disasters [7]. However, with the development and generalized application of the big data industry, the microseismic system is no longer just the processing of microseismic signals is required, and it is required to be used as a sensing device in mine production. As a sensing device, higher requirements for microseismic signal processing are required, and the collected signals need to be more finely divided to ensure effective monitoring of microseismic signals in mines.
For the feature extraction of mine microseismic signals, experts at home and abroad have done a lot of research, mainly including EMD decomposition and timefrequency transform decomposition based on two types of decomposition methods. Among them, the EMD decomposition is the empirical modulus EMD decomposition proposed by Huang [9] et al., which constructs complex nonlinear models into multiple intrinsic moduli for further research and provides certain ideas for the follow-up. Shang et al. [10] studied the construction of IMF components after EMD decomposition of the microseismic signal, matrix, and singular value decomposition and then used the decomposition class with SVM to realize the distinction between microseismic signal and blasting signal. In terms of time-frequency analysis, Zhao et al. [11] used frequency-slicing wavelet transform to study the time-frequency characteristics, frequency band energy distribution, and correlation coefficients of 
microseismic signals and provided a feature recognition method. Tang et al. [12] proposed the use of wavelet energy spectrum coefficients to analyze the energy distribution characteristics of coal and rock fractures and noise signals, which laid an important foundation for the analysis of the energy characteristics of coal and rock fracture microseismic signal modes. Allmann et al. [13] found that the main frequency of the microseismic signal of different causes was differentiated after the $S$ transform of the microseismic signal of the mine. After solving the wavelet packet energy of the microseismic signal and the blasting signal, Zhu et al. [14] found that the energy distribution of the blasting vibration signal and the microseismic signal is different due to the difference in energy distribution and proposed a new idea for the classification of the microseismic signal. However, in the abovementioned documents, most of them distinguish between microseismic signals and blasting signals, and fail to effectively analyze the signals of other events.

In recent years, deep learning methods for fusing time series data and signals have gained widespread attention $[15,16]$, mainly due to the rapid development of the big data industry. The fusion of multiple data features is the essence of integrated feature fusion. Different algorithms are used to merge $[17,18]$ to generate new feature data, which is easier to process [19]. Fusing big data can be classified into three types: data fusion, feature fusion, and decision fusion. Feature fusion is a valuable technique for improving the apparentness of features. The purpose of this paper is to analyze the feature fusion processing of the microseismic signal using the singular value of wavelet packet capacity energy ratio and the component singular value of EMD energy using the unsupervised learning algorithm of deep learning.

\section{Microseismic Signal and Its Digital Features}

2.1. Wavelet Packet Energy Features. Microseismic signals [4] can be expressed by wavelet transformation, as a new method of processing them, by stretching the mother wavelets of finite length or by fast attenuation. The formula is as follows:

$$
\begin{aligned}
X(a, b) & =\frac{1}{\sqrt{b}} \int_{-\infty}^{\infty} x(t) \psi\left(\frac{t-a}{b}\right) \mathrm{d} t X(a, b) \\
& =\frac{1}{\sqrt{b}} \int_{-\infty}^{\infty} x(t) \psi\left(\frac{t-a}{b}\right) \mathrm{d} t,
\end{aligned}
$$

where $X(a, b)$ is the transformed wavelet, $x(t)$ is the original wave signal, $a$ indicates the translation amount, $b$ represents the wavelet scale, and $\psi(t-a / b)$ is the wavelet. To decompose microseismic signals into low- and high-frequency subsignals, the high- and low-frequency components of the wavelet transform are applied simultaneously, and the original signals are mapped on the frequency band to generate corresponding subsignals. According to Parseval's theorem, the signal energy $E_{i}$ decomposed into the $i^{\text {th }}$ subband can be expressed as follows:

$$
E_{i}=\sum_{n=1}^{t} w_{i}(n)^{2} .
$$

Then, the total signal energy $E$ can be obtained as follows:

$$
E=\sum E_{i}
$$

Finally, the energy percentage vector $p$ of each frequency band can be obtained as follows:

$$
p=\left(\frac{E_{i}}{E} \mid i=1,2,3, \ldots, 2^{n}\right) .
$$

2.2. SVD Features of Rock Rupture Signal EMD. The EMD technique was conceived by N. E. Huang and NASA for nonlinear nonstationary signals. The purpose of EMD is to separate a nonlinear nonstationary signal into several independent components, expressed as follows:

$$
x(t)=\sum_{i=1}^{n} \mathrm{IMF}+r_{n},
$$

where IMF is the empirical modulus after multiple decomposition and $r_{n}$ is the residual after this decomposition. For the decomposed quantities, the empirical modulus matrix (6) is constructed as follows:

$$
\mathrm{IMF}=\left(\mathrm{IMF}_{1}, \mathrm{IMF}_{2}, \ldots \mathrm{IMF}_{i}\right)^{T} .
$$

IMF of the empirical modulus matrix is decomposed to obtain the singular value vector $v$ as shown in the following formula:

$$
v=\left(\sigma_{1}, \sigma_{2}, \ldots, \sigma_{i}\right)
$$

where $\sigma$ is the singular value after decomposition.

\section{Mine Microseismic Signal Feature Fusion Method Based on Fully Connected Neural Network}

3.1. Unsupervised Learning-Based Feature Fusion Method. In unsupervised learning, two steps are performed: encoding and extracting data features and subsequently decoding and inverting the extracted features. Fusing features extracted through feature extraction are the next step. As shown in Figure 1, there is a schematic diagram showing the unsupervised learning method.

Figure 1 shows the unsupervised learning neural network illustrated by $k, \mathrm{~L} 1, \mathrm{~L} 2, \mathrm{~L} 3$, and L4 coding structures, L5, L6, L7, and L8 decoding inversion structures, $k_{\text {new }}$ is the extracted feature vector, and $k^{\prime}$ represents the generated data after coding. As the neurons gain weight, the dark color is representative of values with high weight and the light color is indicative of values with low weight. During the training process, $k$ is compared with $k$ ' in order to determine the structure of the network. 


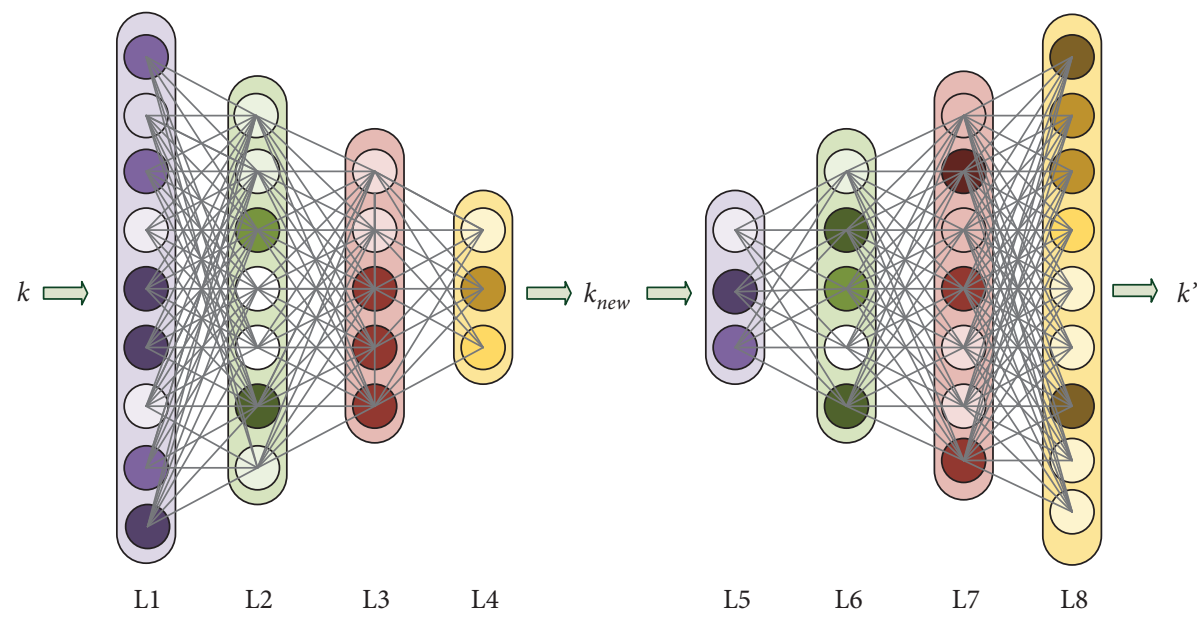

FIgURE 1: Schematic diagram of the unsupervised learning algorithm.

3.2. M-W\&E Feature Fusion Method. In this paper, we present a digital fusion method that can be applied to the feature fusion of mine seismic signals. In this method, certain subfrequency bands are mapped by an energy vector $P$ after the wavelet transformation with an empirical modulus matrix $v$.

(1) To generate the wavelet packet energy percentage vector $p$ and singular value vector $v$ from the mine microseismic signals, the energy ratio solution of the wavelet packet transform and singular value decomposition of the empirical modulus are applied.

(2) Combining the wavelet packet energy percentage vector $p$ and the singular value vector $v$ in the first dimension to reduce the symbol size in (8), the formula for constructing the fusion vector $k$ is shown as follows:

$$
k=(p, v)
$$

(3) The process of integrating signals $k$ after they have been entered into the unsupervised learning feature neural network produces a new fusion feature $k_{\text {new }}$. The overall flow chart of the feature fusion algorithm is shown in Figure 2.

\section{Key Parameters Selection and Structure Construction}

4.1. EMD Experience Modulus Decomposition Layers. According to Shang et al. [10], the singular value division of microseismic signals of mines and blasting signals points to a distinct difference between blasting vibration signals and rock rupture signals. Figure 3 shows the result of EM decomposition of the rock rupture signal.

Figure 3 shows that this is the case in which the typical mine microseismic signal can be composed of $10 \mathrm{IMF}$ empirical moduli. When the empirical modulus is decomposed through IMF4 and 5, the correlation between the empirical modulus and the microseismic signal changes significantly. The empirical modulus has only very limited relation to the microseismic signal in the case of IMF6, suggesting that we can gain fewer data from it. As shown in Table 1, there were the following empirical decomposition moduli.

Decomposition layers are listed in Table $1 ; 14$ is the maximum, and 8 is the minimum number. Microseismic signals with ten or eleven layers typically exhibit a decomposition modulus of ten or eleven, and $79.02 \%$ of mine microseismic signals have ten or eleven layers. As a result, utilizing only one layer of empirical modulus decomposition of the microseismic signal will not distinguish the microseismic signals, whereas utilizing more layers will result in a large amount of irrelevant information interfering with the singular value. Thus, it is necessary to establish and analyze the number of empirical modulus decomposition layers of microseismic waves. An important evaluation tool is the correlation coefficient [17], which can reflect the degree of information in the IMF component containing the original microseismic signal after the microseismic signal decomposition process. A correlation coefficient is calculated using the following formula:

$$
c\left(x, \mathrm{IMF}_{i}\right)=\frac{\sum_{n=1}^{t}(x(n)-\bar{x})\left(\mathrm{IMF}_{i}(n)-\overline{\mathrm{IMF}_{i}}\right)}{\sqrt{\sum_{n=1}^{t}(x(n)-\bar{x})^{2} \sum_{n=1}^{t}\left(\mathrm{IMF}_{i}(n)-\overline{\mathrm{IMF}_{i}}\right)^{2}}},
$$

where $c\left(x, \mathrm{IMF}_{i}\right)$ is the correlation coefficient, $x(n)$ is the mine microseismic signal, and IMFI is the number of EMD decomposition layers. After decomposition, the number of each IMF component, the correlation coefficient of the original signal as well as the correlation coefficient calculation results were plotted, as shown in Figure 4.

IMF7 decomposition is shown in Figure 4 as an important boundary. Decomposition of the IMF7 signal shows that before general correlation coefficient is relatively high, but after general correlation coefficient is reduced to less than 0.2 ; this shows the weak correlation with the original signal. For 4 8 layers of IMF components, singular value vectors of each type were decomposed and the correlation 


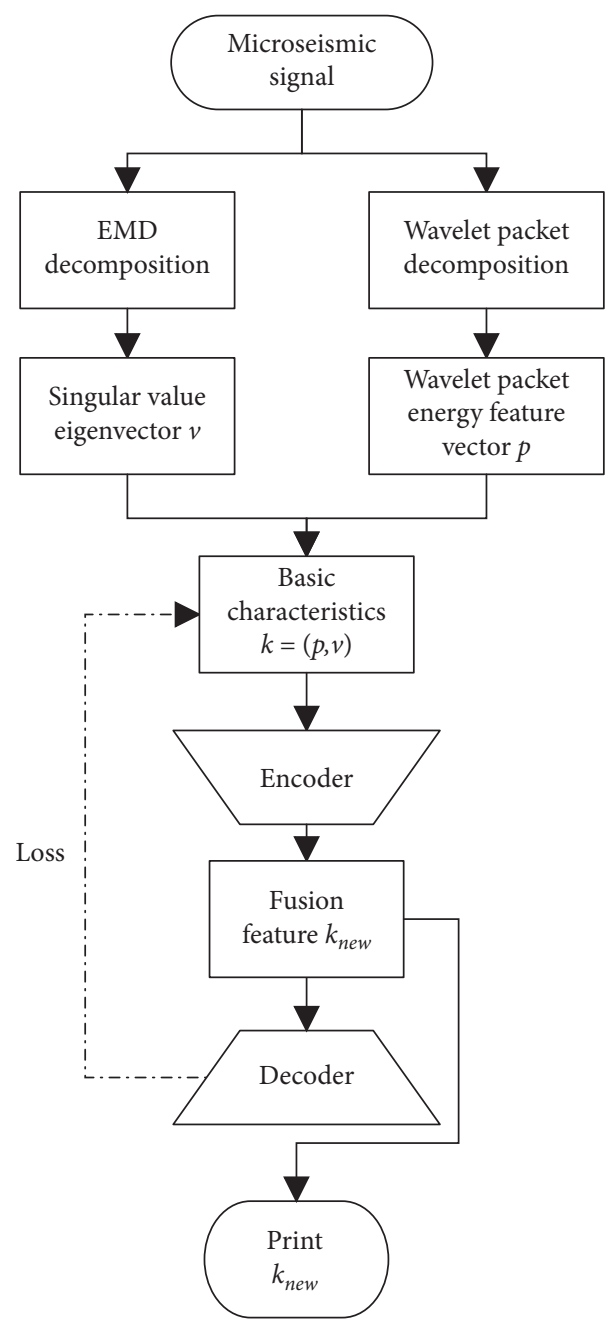

FIgURE 2: M-W\&E feature fusion method flowchart.
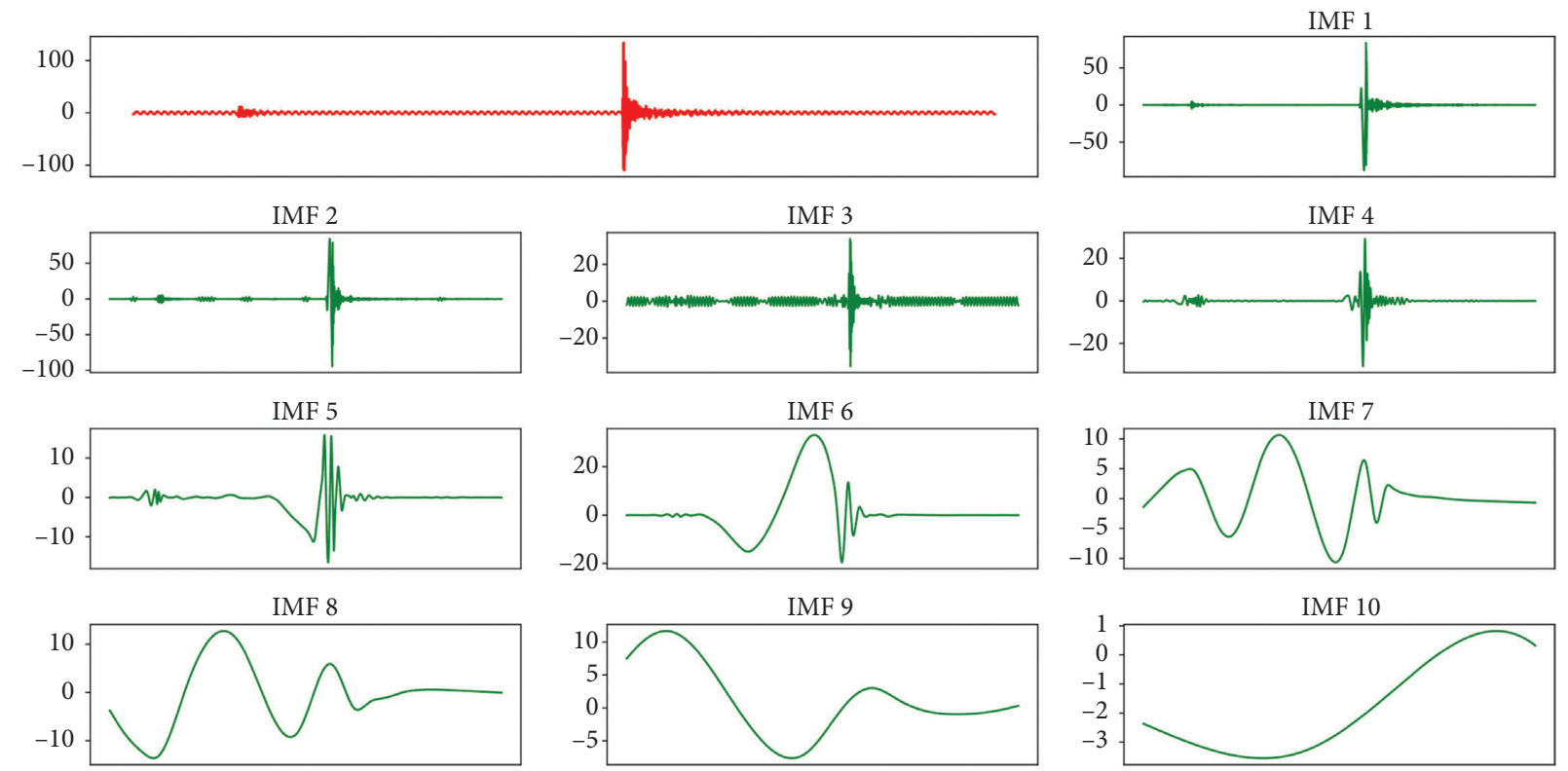

IMF 9
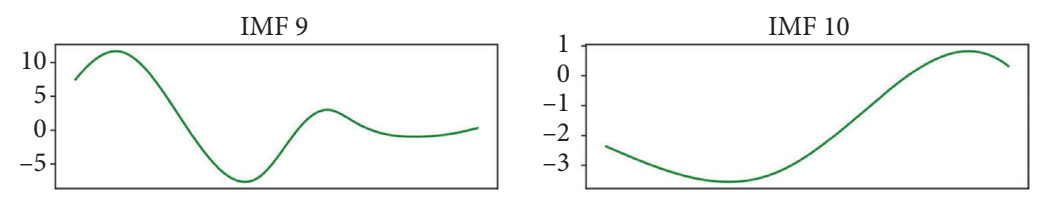

FIGURE 3: Decomposition of the empirical modulus of rock fracture signal. 
TABLE 1: Distribution of IMF decomposition layers of each microseismic signal.

\begin{tabular}{lccccccc}
\hline Decomposition layer & 8 & 9 & 10 & 11 & 12 & 13 & 14 \\
\hline Number of groups & 5 & 70 & 227 & 244 & 37 & 10 & 3 \\
\hline
\end{tabular}

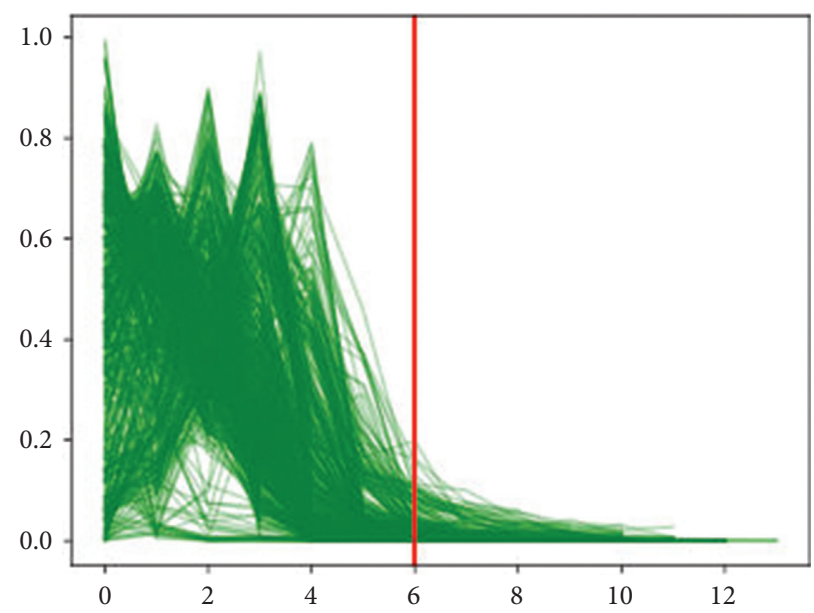

Figure 4: Correlation coefficient between IMF classification and original microseismic signal.

between the types of singular value vectors was analyzed. Then, the mean correlation was calculated according to the following calculation:

$$
r_{k}=\frac{1}{n(n-1)} \sum_{i=1}^{n} \sum_{j=1}^{n-1} c\left(E_{i}, E_{j}\right),
$$

where $r_{k}$ is the mean correlation coefficient of the decomposition layer $k$ and $E_{i}$ and $E_{j}$ are the energy ratios of different types of wavelet packets in the layer $k$ decomposition, $i \neq j$. The mean correlation coefficients are shown in Table 2.

4.2. Number of Wavelet Decomposition Layers. As the number of decomposition layers increased following the EMD_SVD, the correlation coefficients of the singular value vectors decreased with an increasing number of IMF components, but they did not change much with the increase in IMF components. Thus, the IMF component was determined to be 6 in this feature fusion.

After wavelet packet transformation, Zhu et al. [14] hypothesized that the energy band distribution of rock fracture microseismic signals differs from that of blasting signals. Figure 5 shows the original waveform signals and the decomposition of the rock rupture signal using four layers of wavelet packets.

As illustrated in Figure 5, the aaaa subwavelet diffuses noise signals with a frequency band of $0 \sim 31.25 \mathrm{~Hz}$. In addition, after the decomposition of mine microseismic signals showed a sharp decrease in peak and valley values, mainly concentrated in the wavelets ended with ad and dd, it shows that the detailed features of the microseismic signal can be amplified at the time of the final layer analysis and can be distinguished by calculating the energy percentage vector $p$ of each frequency band. It is necessary to divide the number of
TABLE 2: Singular value vector correlation coefficients of various signals.

\begin{tabular}{lccccc}
\hline Decomposition layer & 4 & 5 & 6 & 7 & 8 \\
\hline$r_{k}$ & 0.5152 & 0.4556 & 0.3257 & 0.3234 & 0.3143 \\
\hline
\end{tabular}

layers of decomposition before calculating vector. Zhu et al. [4] found that after decomposition twice by wavelet packets, the microseismic signals had quite different distributions because of the different dominant frequencies. The wavelet decomposition layer count is another important parameter for the $\mathrm{M}-\mathrm{W} \& \mathrm{E}$ feature fusion. The number of microseismic signal layers based on the distribution of $E_{-} w$ energy frequency band after the 3rd, 4th, 5th, and 6th wavelet packet layers was determined. The energy distribution diagram of signals of various channels is shown in Figure 6.

Figure 6 shows that most microseismic signals have their main energy concentrated in the relatively low-frequency range, while blasting vibration signals (green) have their energy distributed over high and low frequencies. The energy decomposition is almost exclusively in the first layer at the 3rd layer, but after the 6th layer, it shows a number of different characteristics. There is a clear distinction between noise-disturbed low-frequency band and signal-disturbed low-frequency band for hand coal (yellow), shovel coal (blue), and hoe coal (red). A six-layer wavelet packet decomposition accounts for $70 \%$ of coal rock after knock-on (purple), 55\% after rupture (brown), and $40 \%$ after mine car passing by (pink) after the third, fourth, and fifth layers of wavelet packet decompositions. By summing the mean correlation coefficients among waveforms in Figure 6 and their differences between one another, one can calculate the correlation coefficient under each layer decomposition. For different wavelet packet energy ratios, the calculated results are shown in Table 3. 


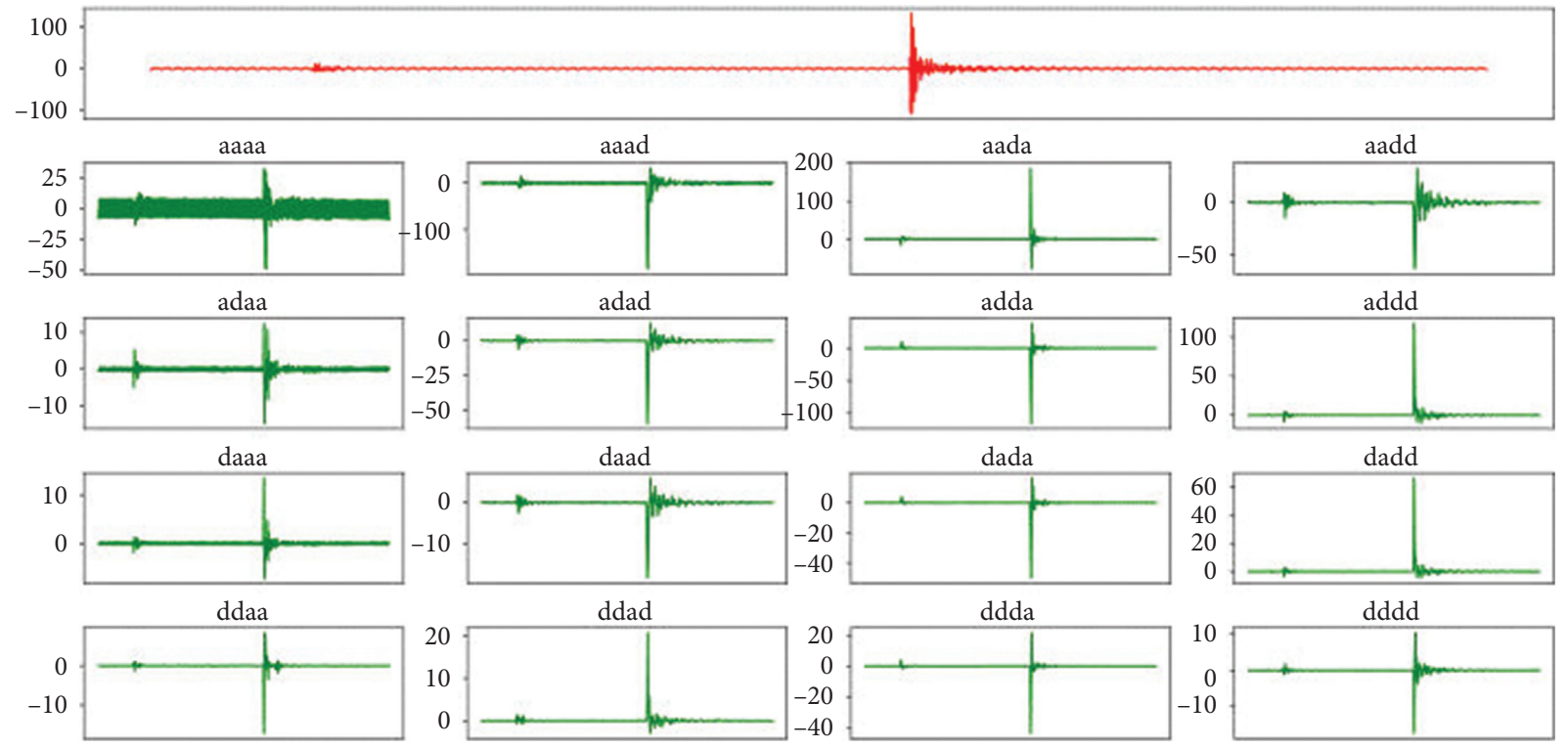

FIGURE 5: Wavelet packet decomposition of rock fracture signal.

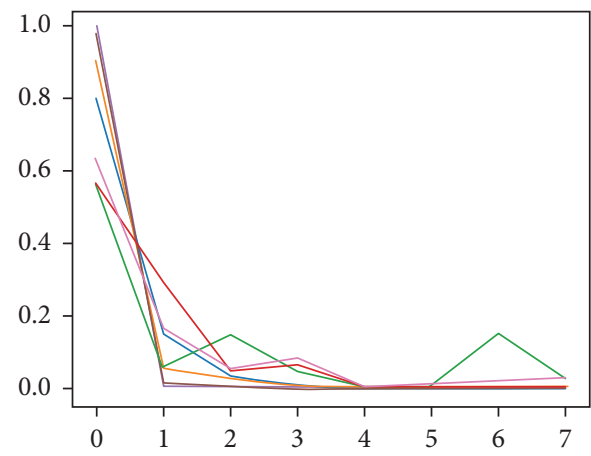

(a)

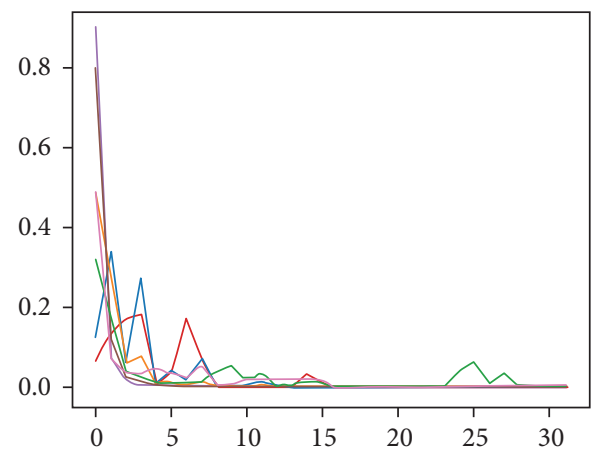

(c)

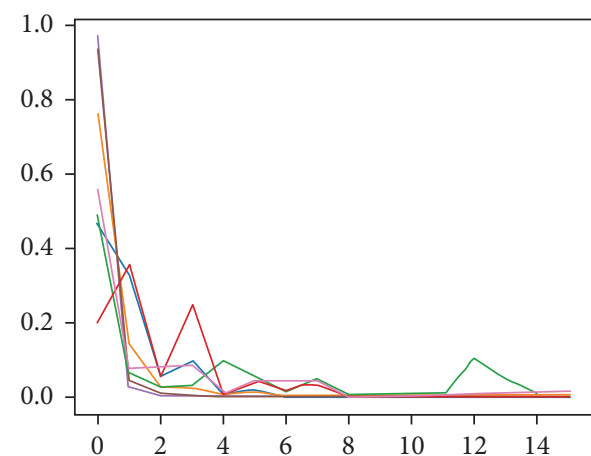

(b)

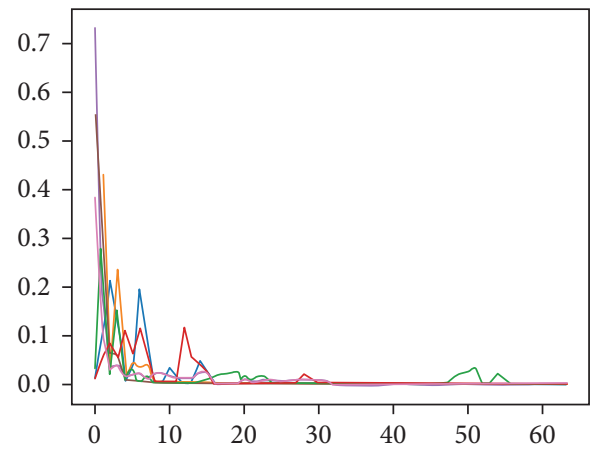

(d)

Figure 6: Frequency band energy ratio of each decomposition layer of microseismic signal: (a) 3-layer wavelet packet analysis; (b) 4-layer wavelet packet analysis; (c) 5-layer wavelet packet analysis; (d) 6-layer wavelet packet analysis.

The mean correlation coefficient for each layer of decomposition decreases significantly with the gradual increase in the number of layers, showing that the features are clearly differentiated. Wavelet packet energy decomposition is completed after the sixth layer, and the mean correlation coefficient returns to a stable state. After taking into account the energy correlation coefficient and the calculation amount in this paper, we adopt 6 wavelet packet 
TABLE 3: Correlation coefficient of energy proportion of each decomposition layer wavelet packet.

\begin{tabular}{lcccccc}
\hline Decomposition layer & 3 & 4 & 5 & 6 & 7 & 8 \\
\hline$r_{k}$ & 0.9127 & 0.7958 & 0.6620 & 0.4620 & 0.4578 & 0.4563 \\
\hline
\end{tabular}

decomposition layers based on the quantity to be calculated increasing significantly after the wavelet packet decomposition at a high layer.

\subsection{Structure of Unsupervised Learning Neural Network.} For feature fusion, the structure of unsupervised learning neural networks is important. It is possible that improperly selecting the neural network may have an impact on the feature, as well as cause the extraction results to be poor. To accomplish unsupervised learning, this paper builds a neural network. In VGG, a neural network structural model, the spatial scale of one layer will be reduced by one fourth of that of the highest layer due to pooling layers during feature extraction layer by layer [16]. A fusion feature extraction model including only two layers is presented in this paper as well as the feature dimension after extraction. A standard feature extraction model is examined using the VGG neural network structure. The four-layer neural network structure model in Figure 7 provides clustering images with different output feature lengths.

The increased output dimension is evident in Figure 7 where the feature changes significantly. A clustering effect is better when output features have a length of 8 than when features have three or four dimensions. A classification type consists of seven attributes. The class types are represented through three dimensions $-4,6$, and 10 , and the dimensions are hard to express because the attribute dimensions with outputs of 4 and 6 are smaller than the classification types. After 10 dimensions, the output dimensions have become more than the classification types, leading to an interference dimension and increased t-SNE size reduction times, thus blurring boundaries, but the 10 dimension contains more information, and the boundary definition is more comfortable than the 4 or 6 dimensions. A neural network structural model outputs an output feature of 8 . Feature output length of 8 was used in the neural network model. Following the analysis of the number of learning layers, the linkage between the neural network structure model with the 8 output feature was calculated using different layers and the neural network structure model. The output result is shown in Table 4.

The average linkage shows a trend of initially rising and then reducing with the increase in feature extraction layers, as shown in Table 4. With higher average linkage, it is easier to distinguish between different types of signals. Accordingly, 4 neural network layer outputs are selected, with each output layer using a sigmoid activation function. The detailed structure of each linear layer is shown in Table 5 .

\section{Example Verification}

5.1. Analysis of Typical Field Data. An analysis of the microseismic signals collected from the mine working faces in Shanxi Province is presented in this paper. Currently, the coal mine works with an initial working surface of 10\#, inclination of $0^{\circ} \sim 8^{\circ}$, thickness of $2.58 \sim 2.62 \mathrm{~m}$, average thickness of $2.6 \mathrm{~m}$, hardness coefficient of $f=0.9 \sim 1.1$, and mudstone roofs around soft rock. Embedded in this system is a microseismic sensor with a buried depth of $25 \sim 50 \mathrm{~m}$ and sampling frequencies of $1 \mathrm{KHz}$.

Figure 8 shows that the rock rupture microseismic signal No. 4 and the blasting vibration signal No. 5 show some similarities to the extent that their tail waves gradually diminish, but that the blasting vibration signal has higher amplitude than the rock rupture signal. Rock fracture signals have an amplitude similar to EMI signals, but the EMI signal appears chaotic and spiky. On the waveform diagram, all three types of manual operation signals 0,2 , and 7 could be seen as "high-low-high" patterns as they were generated manually by humans. An analysis of the above types of signals using feature fusion was carried out for the purpose of finding a method that will be easier to identify.

5.2. Feature Fusion Calculation. Figure 8 shows the results of the M-W\&E fusion for 8 types of mine microseismic signals. To display the fusion of microseismic signal features, 2 groups of each type of microseismic signal were selected. As shown in Table 6, the values of the features after fusion are displayed. Based on Table 6, the two fused signals of $k_{\text {new1 }}$ and $k_{\text {new }}$ outperform the other features after fusion in dimension learning. It is apparent that different signals occupy smaller, special ranges in the same dimension of the feature, which implies that the neurons have mastered the different features at varying degrees in the learning process. There is a large difference between the signal types in $k_{n e w 1}$, especially in the ranges occupied by the signals.

5.3. Evaluation of Fusion Results. Analysis and comparison of the M-W\&E data fusion algorithm were conducted using wavelet packet energy and EMD_SVD. To evaluate the advantages and disadvantages of feature fusion, the twodimension spatial visualization of the three types of features and the SVM classification results use t-SNE spatialvisualization evaluation method to compare.

$\mathrm{t}-\mathrm{SNE}$ is a manifold nonlinear dimensionality reduction visualization method that allows the viewer to see the feature extraction effects of neural network models by visually reducing the dimensionality of their data. Consequently, the present paper uses the $\mathrm{t}$-SNE process visualization technique to reduce the high-dimensional features obtained from after the convolutional layer to a two-dimensional plane space and to examine their performance capability. An analysis of dimension reduction and visualization was performed on the original signal, the wavelet packet fusion signal, the EMD fusion signal, and the proposed feature fusion algorithm in this paper. The results are shown in Figure 9. 


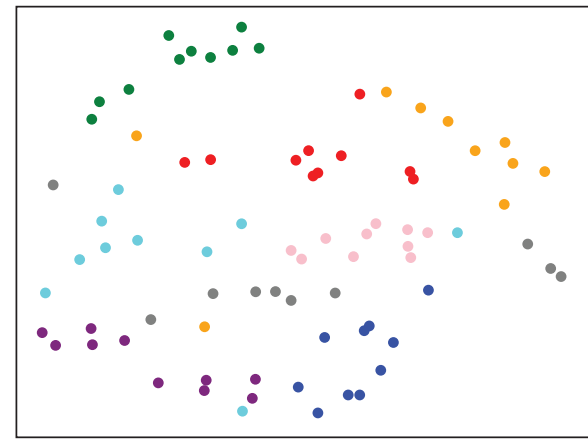

(a)

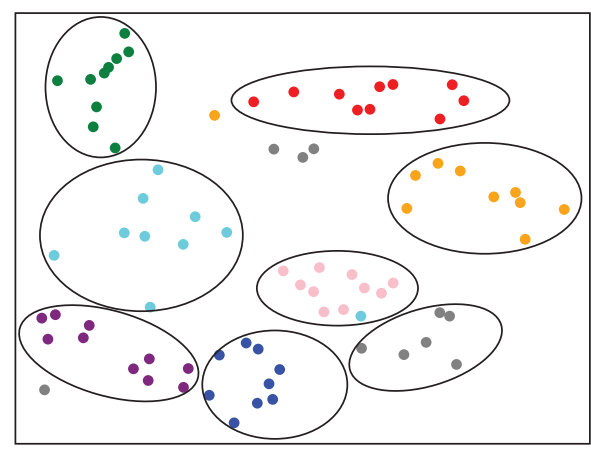

(c)

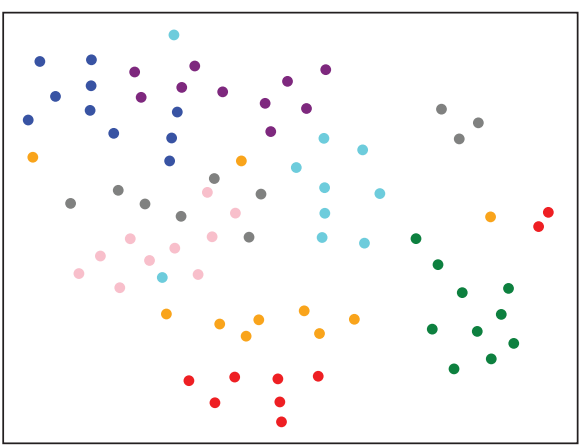

(b)

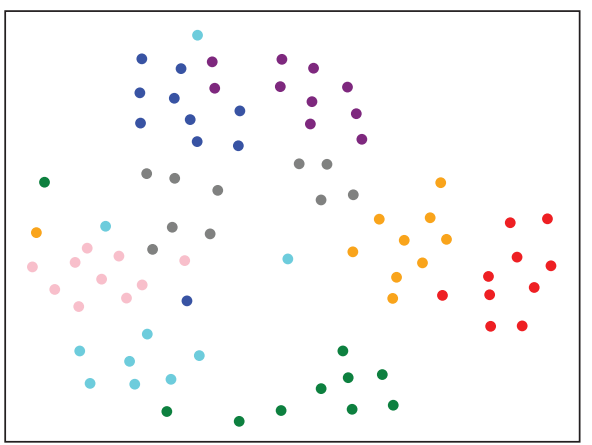

(d)

FigURE 7: Cluster graph of each feature length under the 4-layer feature extraction layer: (a) 4 layers 4 dimensions; (b) 4 layers 6 dimensions; (c) 4 layers 8 dimensions; (d) 4 layers 10 dimensions.

TABLE 4: Average linkage of different feature extraction layers.

\begin{tabular}{lr}
\hline Feature extraction layers & Average linkage \\
\hline 3 & 3.475 \\
4 & 4.234 \\
5 & 3.665 \\
6 & 3.398 \\
\hline
\end{tabular}

TABLE 5: Unsupervised learning feature neural network structure parameters.

Feature fusion extraction layer Input parameters Output parameters Feature inversion layer Input parameters Output parameters

\begin{tabular}{lccccc}
\hline L1 & 80 & 40 & L5 & 8 & 10 \\
L2 & 40 & 20 & L6 & 10 & 20 \\
L3 & 20 & 10 & L7 & 20 & 40 \\
L4 & 10 & 8 & L8 & 40 & 80 \\
\hline
\end{tabular}

It can be seen from Figure 9(a) that in the case of direct dimension reduction of microseismic signals without any treatment, the two-dimensional spatial distribution after dimensionality reduction by $\mathrm{t}$-SNE presents a mixed distribution state, while the energy features after wavelet packet decomposition and those after EMD decomposition show a certain clustering property, of which the green one is easy to be recognized and presents clustering either after EMD decomposition or wavelet packet decomposition. Compared with EMD energy decomposition, wavelet packet energy dimension reduction is slightly better in terms of overall performance. The three types of microseismic signals appeared similarly mixed after a wavelet packet energy decomposition, but a wavelet packet energy decomposition produced a relatively clear differentiation among them. In addition, the three microseismic signal features pink, gray, and yellow occupy the same position after dimensionality reduction and visualization, indicating that all three types of microseismic signal features are similar to each other after wavelet packet energy decomposition. In Figure 9(d), all types of microseismic signal features after the fusion algorithm showed obvious clustering effect after $t-S N E$ dimension reduction, but there was a yellow microtremor signal near the dimension reduction range of the red microseismic signal feature at the lower right corner, 

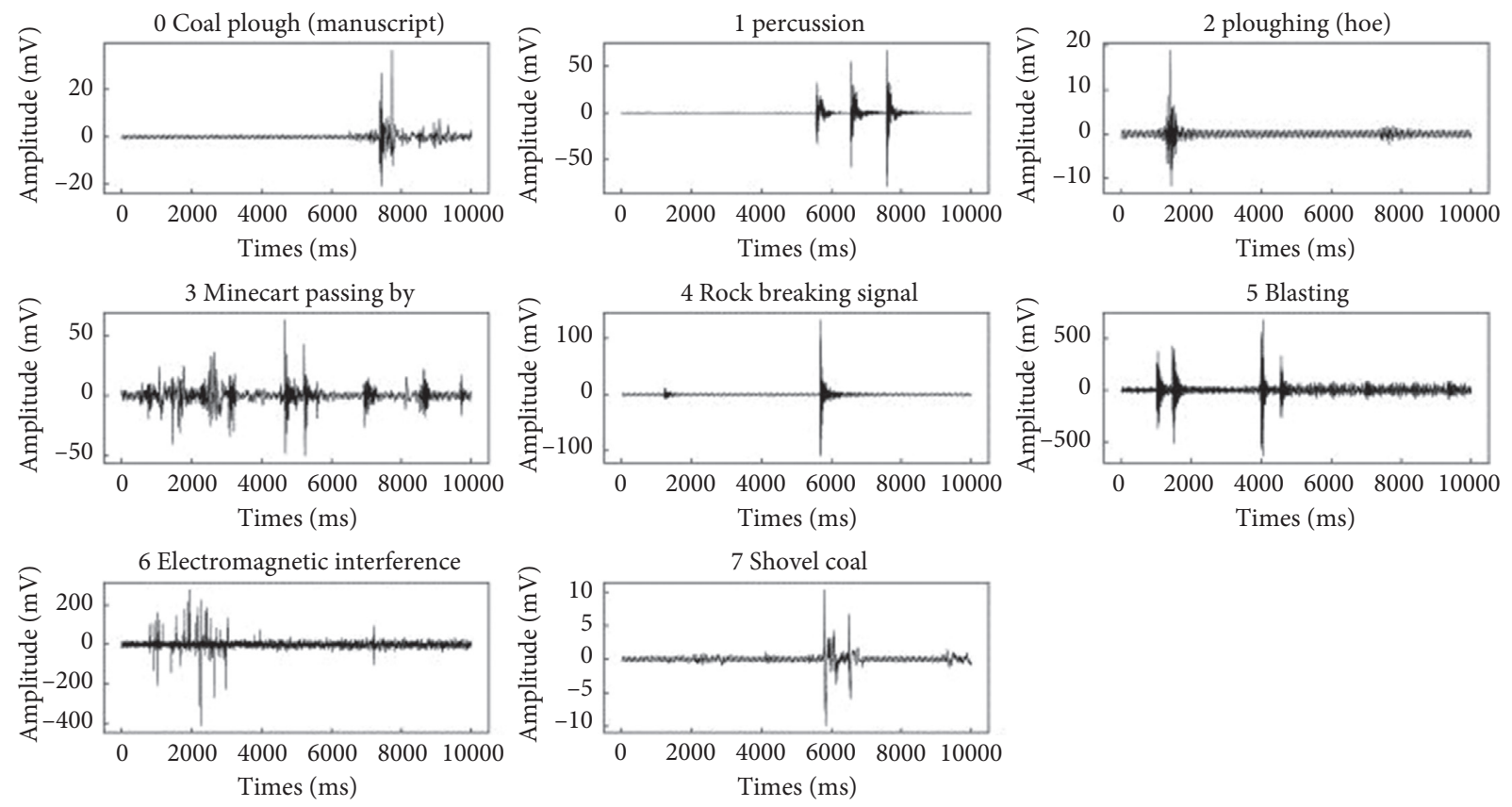

FIGURE 8: Waveform image of microseismic signals in common mines.

TABLE 6: Fusion characteristics of various events.

\begin{tabular}{|c|c|c|c|c|c|c|c|c|}
\hline Event & $k_{\text {new } 1}$ & $k_{\text {new2 }}$ & $k_{\text {new3 }}$ & $k_{\text {new } 4}$ & $k_{\text {new5 }}$ & $k_{\text {new6 }}$ & $k_{\text {new } 7}$ & $k_{\text {new8 }}$ \\
\hline $0 \_1$ & 0.5001 & -1.821 & 0.02581 & -1.325 & 0.2765 & 0.1244 & -0.1289 & 0.1526 \\
\hline $0 \_2$ & 0.5617 & -2.122 & -0.1481 & -1.368 & 0.7622 & 0.1893 & -0.8002 & -0.1864 \\
\hline 1_1 & -0.0343 & 0.7126 & -0.1530 & 0.2844 & 0.1190 & 0.2380 & -0.2050 & -0.1785 \\
\hline $1 \_2$ & 0.08697 & 0.9340 & 0.2391 & 0.4886 & 0.3081 & 1.649 & 1.553 & 0.1097 \\
\hline $2 \_1$ & -0.5977 & -0.3184 & 0.2722 & -0.8219 & 0.4543 & 0.1980 & 0.1859 & -0.4004 \\
\hline $2 \_2$ & -0.7905 & -0.2000 & 0.4418 & -1.647 & 0.9214 & -0.3722 & -0.3227 & -0.2133 \\
\hline 3_1 & 0.3421 & -1.242 & 0.4294 & -0.3219 & -0.3893 & 1.000 & -0.7345 & -0.3691 \\
\hline $3 \_2$ & 0.3479 & -1.018 & 0.03796 & -0.3265 & -0.8431 & 0.7047 & -0.5303 & -0.5500 \\
\hline $4 \_1$ & -0.3321 & -0.8270 & -0.3389 & -0.9336 & 0.1558 & 0.3074 & -0.4282 & -0.2500 \\
\hline $4 \_2$ & -0.3397 & -0.6159 & 0.4481 & -1.538 & 0.2683 & 0.4947 & -0.9815 & -0.4181 \\
\hline 5_1 & -0.1381 & -0.5451 & 0.7698 & -1.989 & 0.2219 & 0.3403 & -0.7560 & 0.3496 \\
\hline $5 \_2$ & -0.1537 & -0.1002 & 1.038 & -1.165 & 0.2116 & 0.3879 & -0.3852 & 0.6888 \\
\hline $6 \_1$ & 0.3253 & 0.009533 & -0.3325 & -0.9649 & 0.8771 & 0.5050 & 0.1364 & 0.2211 \\
\hline $6 \_2$ & 0.3612 & -0.032 & -0.434 & -0.9573 & 0.8680 & 0.5043 & -0.9699 & -0.6800 \\
\hline 7_1 & 0.9409 & -0.9920 & -0.5088 & -0.3364 & -0.9371 & 0.09867 & -0.07757 & 0.1945 \\
\hline 7_2 & 0.7031 & -0.9597 & -0.3833 & -0.5293 & -0.5036 & 0.02525 & -0.3370 & 0.4775 \\
\hline
\end{tabular}

indicating that although the microseismic signal feature fusion method proposed in this paper has optimized the microseismic signal feature extraction to a certain extent, there is still a certain error.

5.4. SVM Classification Evaluation. Eight types of microseismic signals were considered for identification based on the classification evaluation shown in Figure 8. Using the 80 groups of microseismic signals found in the training set, we divided each type of microseismic signal into ten groups. During the testing process, the same component is used as it was in the training set. SVM consists of decomposing the second-type signals into hyperplanes, a classical method [15]. Microseismic signals were classified into eight types using hierarchical support vector machines (H-SVMs). In this method, all types are first divided into two type families, each of which is further divided into two subtypes, and so on. In SVM classification, the radial basis function (RBF) was adopted, which is expressed as shown in formula (11), the penalty parameter of the function is $c=2$, and the kernel parameter is 1 .

$$
K(x, x)=e^{-r\left\|x-x_{i}\right\|_{2}} .
$$

Hierarchical support vector machine was used to train the training set, and the test set was classified. The test results are shown in Table 7.

To facilitate the analysis of the test results, a confusion matrix of classification results of the test set was drawn, as follows.

Following SVM classification, the wavelet packet energy classification effect is shown in Figures 10-12, 


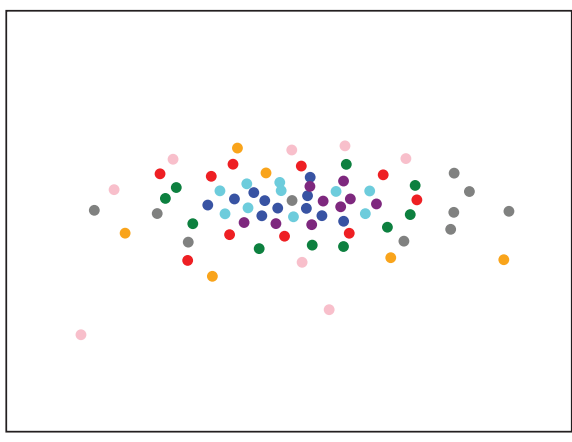

(a)

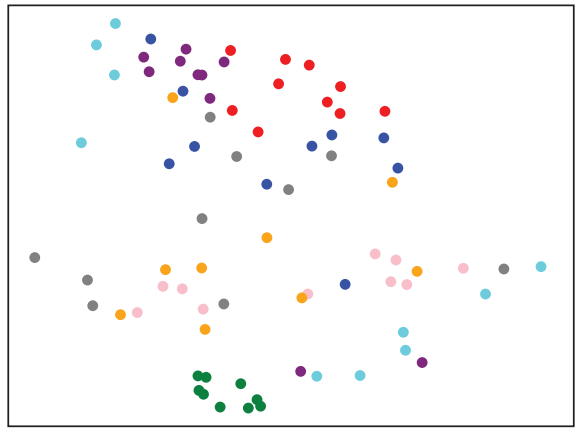

(c)

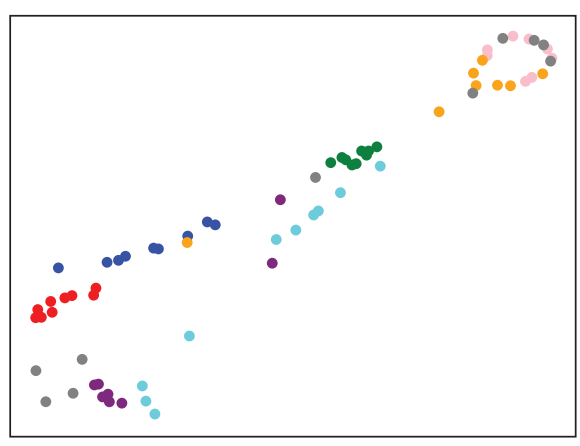

(b)

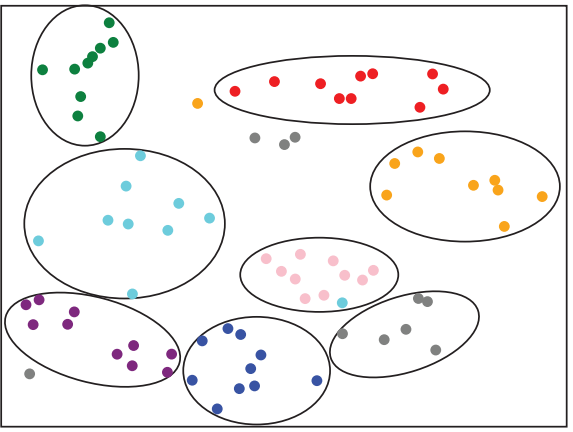

(d)

FiguRE 9: Dimensionality reduction map after various feature extractions: (a) no processing; (b) wavelet packet energy feature distribution; (c) EMD_SVD feature distribution; (d) M-W\&E feature distribution.

TABLE 7: Various features are counted under SVM recognition.

\begin{tabular}{lccc}
\hline Classification features & Times $(\mathrm{ms})$ & Identify the correct number & ACC $(\%)$ \\
\hline Wavelet packet energy & 48.74 & 48 & 60 \\
EMD_SVD & 75.59 & 69 & 86.2 \\
M-W\&E & 217.22 & 74 & 92.5 \\
\hline
\end{tabular}

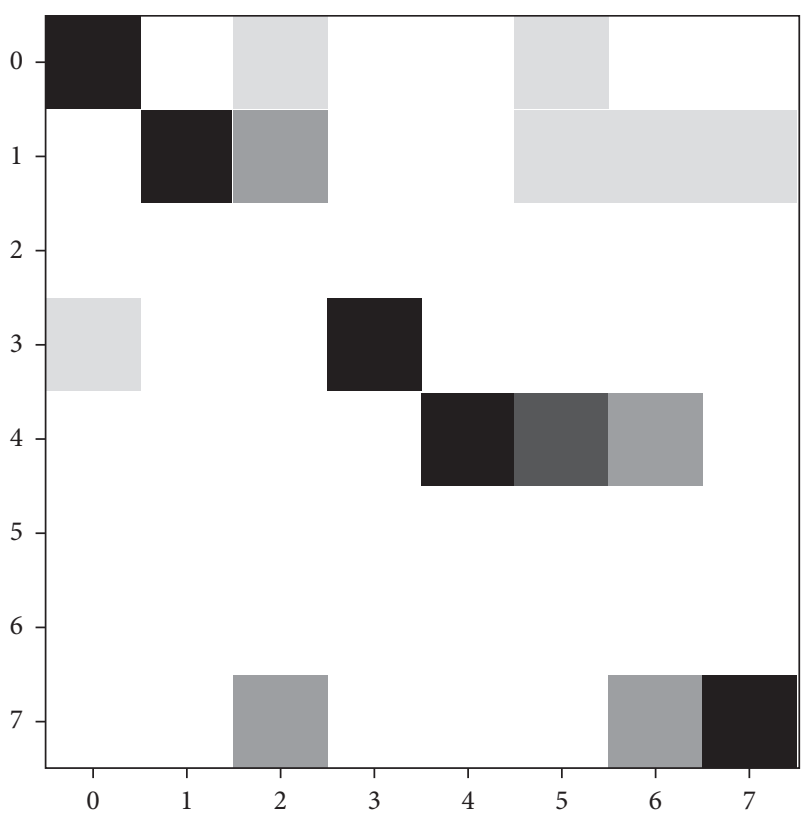

FIGURE 10: Wavelet packet energy feature classification: confusion matrix.

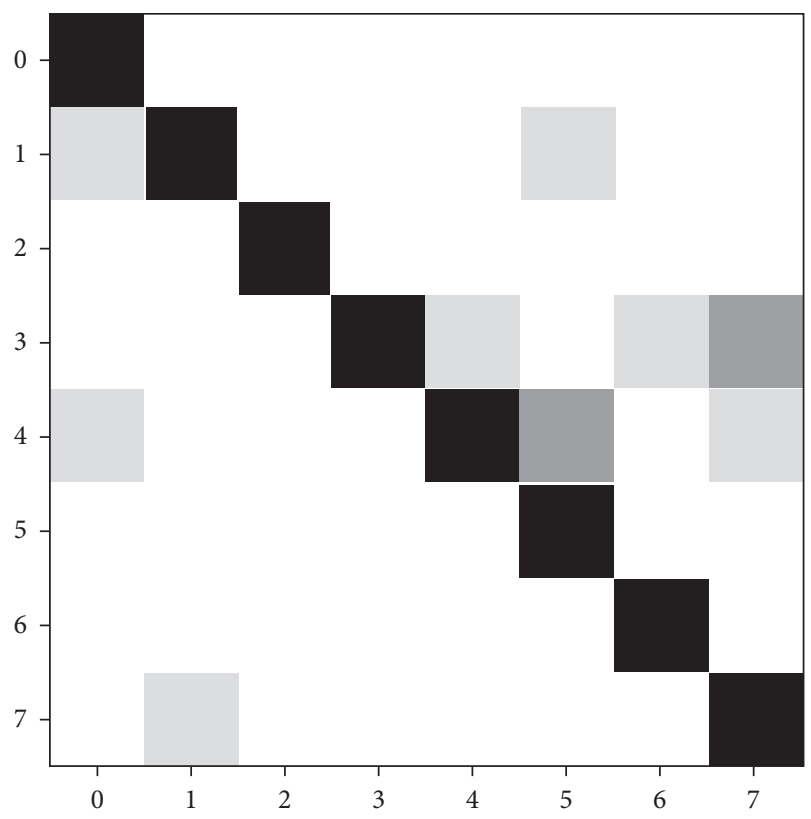

FIGURE 11: EMD_SVD classification confusion matrix. 


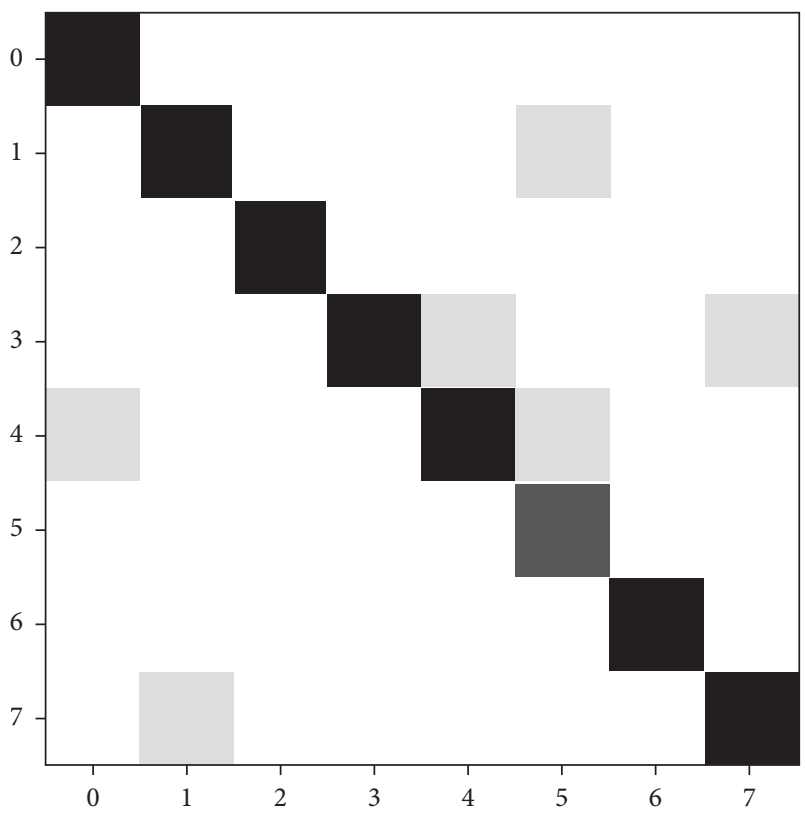

FIGURE 12: M-W\&E feature classification confusion matrix.

which shows only 48 correct predictions, the lowest prediction classification accuracy of all three methods. The fact that the main frequency bands of the energy of Nos. 3, 5, and 6 events were quite similar suggests that the main feature required for multiple classification is the wavelet energy ratio, which is difficult to predict. EMD_SVD significantly improved the classification accuracy, and 69 accurate predictions were made, indicating that the accuracy of classification was high. Moreover, the results of the correct classification are clearly distinguishable from those from other positions on the main diagonal of the confusion matrix. By using EMD_SVD and wavelet packet energy data as criteria for classification, it was possible to ideally combine the strengths of these two techniques, and most importantly, the identifying accuracy of the No. 7 event was significantly enhanced. By comparing SVM classification results with $\mathrm{t}$-SNE classification results, it was found that although the effect of wavelet packet energy after t-SNE dimension reduction and visualization of data is better than that of EMD_SVD, the effect after recognition is worse than that of EMD_SVD feature, indicating that EMD_SVD feature distribution is not suitable for t-SNE dimension reduction expression. However, some identification errors still persist after the M-W\&E method is reduced by $\mathrm{t}-\mathrm{SNE}$, indicating that the data features are complex. In this paper's feature fusion method, there is still room for improvement in small sample learning.

\section{Conclusions}

(1) A multifeature fusion of mine microseismic signals is achieved by combining features from EMD singular values and wavelet packet energy, which is based on the unsupervised learning mode in deep learning.
Microseismic signals are processed in smart mines using this method.

(2) After the M-W\&E model proposed in this paper performs the fusion of the singular value decomposition features of the first 6 layers of EMD and the wavelet packet feature on the mine microseismic signal, the recognition accuracy rate reaches $92.5 \%$, which is higher than the recognition result of the single feature method and basically meets the requirements. The actual needs of the project site also provide some experience for the feature fusion of microseismic signals.

(3) Although in the M-W\&E model, the recognition success rate in the multicategory recognition task is $92.5 \%$, which improves the recognition accuracy to a certain extent, the average time consumption reaches $217.22 \mathrm{~ms}$, which is higher than the cost of the single feature recognition mode. At the same time, we do not yet know how unsupervised learning algorithms interpret features. Thus, the next research direction will be to find ways to reduce the time it takes to do research and to make it easier to interpret [20].

\section{Data Availability}

The data used to support the findings of this study are included within the article.

\section{Conflicts of Interest}

The author declares that there are no conflicts of interest.

\section{Acknowledgments}

This research was financially supported by Research on Dynamic Monitoring and Intelligent Early Warning Technology of Coal and Gas Outburst (Qiankehe Platform Talent [2019]5675).

\section{References}

[1] T. Jin, X. Yang, H. Xia, and H. Ding, "Reliability index and option pricing formulas of the first-hitting time model based on the uncertain fractional-order differental equation with Caputo type," Fractals, vol. 29, no. 1, Article ID 2150012, 2021.

[2] T. Jin, H. Ding, H. Xia, and J. Bao, "Reliability index and Asian barrier option pricing formulas of the uncertain fractional first-hitting time model with Caputo type," Chaos, Solitons \& Fractals, vol. 142, 2021.

[3] Y. Xue, J. Cao, R. Tian, K. Du, and Y. Yao, "Wavelet-based cepstrum decomposition of seismic data and its application in hydrocarbon detection," Geophysical Prospecting, vol. 64, no. 6, 2016.

[4] Q. Zhu, Y. Feng, M. Cai, J. Liu, and H. Wang, "Interpretation of the extent of hydraulic fracturing for rockburst prevention using microseismic monitoring data," Journal of Natural Gas Science and Engineering, vol. 38, pp. 107-119, 2017.

[5] W. Li, Y. K. Liu, and B. J. Liu, "Downhole microseismic signal recognition and extraction based on sparse distribution features," Chinese Journal of Geophysics, vol. 59, no. 10, 2016. 
[6] N. Xu, C. Tang, S. Chun, and Z. Liang, "Microseismic monitoring system establishment and its engineering applications to left bank slope of Jinping I Hydropower Station," Chinese Journal of Rock Mechanics and Engineering, vol. 29, no. 5, pp. 915-925, 2010.

[7] M. K. A. Wahed, M. Al Heib, and G. Senfaute, "Mininginduced seismicity: seismic measurement using multiplet approach and numerical modeling," International Journal of Coal Geology, vol. 66, no. 1, pp. 137-147, 2006.

[8] S. C. Maxwell, J. Rutledge, R. Jones, and M. Fehler, "Petroleum reservoir characterization using downhole microseismic monitoring," Geophysics, vol. 75, no. 5, 2010.

[9] N. E. Huang, Z. Shen, S. R. Long et al., "The empirical mode decomposition and the Hilbert spectrum for nonlinear and non-stationary time series analysis," Proceedings of the Royal Society of London. Series A: Mathematical, Physical and Engineering Sciences, vol. 454, pp. 903-995, 1998.

[10] X. Y. Shang, X. Li, K. Peng, and L. Dong, "Feature extraction and classification of mine microseism and blast based on EMD_SVD," Chinese Journal of Geotechnical Engineering, vol. 38, no. 10, pp. 1849-1858, 2016.

[11] G. Y. Zhao, Q. L. Deng, and J. Ma, "Analysis and recognition of mine microseismic signals based on FSWT time-frequency analysis," Chinese Journal of Geotechnical Engineering, vol. 37, no. 2, pp. 306-312, 2015, (in Chinese).

[12] S. F. Tang, M. M. Tong, Y. X. Pan, and X. He, "Energy spectrum coefficient analysis of wavelet features for coal rupture microseismic signal," Chinese Journal of Scientific Instrument, vol. 32, no. 7, pp. 1522-1527, 2011, (in Chinese).

[13] B. P. Allmann, P. M. Shearer, and E. Hauksson, "Spectral dqbes," Bulletin of the Seismological Society of America, vol. 98, no. 4, pp. 2073-2079, 2008.

[14] Q. J. Zhu, F. X. Jiang, Y. M. Yin, X. Z. Yu, and J. L. Wen, "Classification of mine microseismic events based on waveletfractal method and pattern recognition," Chinese Journal of Geotechnical Engineering, vol. 34, no. 11, pp. 2036-2042, 2012, (in Chinese).

[15] B. Luo and E. R. Hancock, "Structural graph matching using the EM algorithm and singular value decomposition," IEEE Transactions on Pattern Analysis and Machine Intelligence, vol. 23, no. 10, pp. 1120-1136, 2001.

[16] G. Wang, Z. Luo, X. Qin, Y. Leng, and T. Wang, "Fault identification and classification of rolling element bearing based on time-varying autoregressive spectrum," Mechanical Systems and Signal Processing, vol. 22, no. 4, pp. 934-947, 2008.

[17] L. Ai, J. Wang, and R. Yao, "Classification of parkinsonian and essential tremor using empirical mode decomposition and support vector machine," Digital Signal Processing, vol. 21, no. 4 , pp. 543-550, 2011.

[18] K. Simonyan and A. Zisserman, "Very deep convolutional networks for large-scale image recognition," 2014, https:// arxiv.org/abs/1409.1556.

[19] V. Vapnik, The Nature of Statistical Learning theory, Springer, N Y, USA, 1995.

[20] R. V. Allen, "Automatic earthquake recognition and timing from single traces," Bulletin of the Seismological Society of America, vol. 68, no. 5, 1978. 